Received: 26 March 2019

Accepted: 22 May 2019

Published online: 31 May 2019

\section{Constructing a three-dimensional graphene structure via bonding layers by ion beam irradiation}

\author{
Mohammad Ali Abdol ${ }^{1}$, Sadegh Sadeghzadeh $\mathbb{1}^{2}$, Maisam Jalaly ${ }^{3}$ \& \\ Mohammad Mahdi Khatibi ${ }^{4}$
}

In recent years, the use of the multilayer graphene sheets has been considered more than the singlelayer due to the cost-effectiveness and the possibility of mass production. But this type of graphene has some kind of structural weakness due to the weak physical link between its layers. Then, in order to strengthen, many structural modifications are proposed by various techniques to manage the mechanisms at interlayer distances. In this study, the focused ion beam irradiation method has been examined to cross-link and strengthen multi-layer graphene sheets with the help of the molecular dynamics simulation technique. Then, uniaxial and transverse tensile tests were performed to check the mechanical properties of obtained cross-linked multilayer graphene sheets. The results of this research can be considered for the creation of a new class of graphene structures. Such structures could be implemented as a membrane in water desalination or as a storage foam in hydrogen or carbon dioxide storage.

The increasing population and global concerns for safe water supply, the global warming due to the burning of non-renewable energy sources and the scarce resources from fossil fuels, all have made water and energy two of the most challenging issues of the present century. In order to overcome the leading environmental crises, there has been an increasing effort to discovery, design and invent more advanced materials to store renewable energy and provide potable water. In the last decade, graphene and its derivative structures have attracted the attention of researchers in various fields of science, due to their exceptional properties. Graphene-related studies are not unique to the single layer of this material and include a wide range of multilayered, composite, and hybrid structures of this material. One of the many types of these structures, which has a lot of potential in many fields, is multi-layered graphene with transverse bonds. In this unique material, the existence of transverse covalent bonds, in addition to strengthening the structure of graphene, is a three-dimensional structure with high porosity and high specific area. Until now, several factors have been proposed to create graphene framework structures, such as boronic acids ${ }^{1}$, diamine monomers ${ }^{2}$, aldehydes ${ }^{3}$, polypropylene imine $e^{4}$, iodobenzene ${ }^{5}$ and diisocyanates ${ }^{6}$. With their placement in the graphene sheets and the connection with a surfactant, three-dimensional structures with intermediate spaces and a high surface area could be constructed. Recently, researchers have succeeded in controlling the intermediate space of graphene layers and filling the distance with epoxy and completely fixing it without using the columnar agent and only by controlling the moisture content. This controlled intermediate space between the graphene plates can selectively pass through water molecules and prevent the transfer of ions in the water ${ }^{7}$. Such a structure is an ideal membrane in the field of desalination and water purification. In the meantime, we see theoretical structures that have not been synthesized yet, such as pillared graphene, which was introduced by Dimitrakakis et al., in $2008^{8}$, a structure consisting of single layers of graphene bonded by carbon nanotubes. By the simulations, it has been proven that the doped type of this material with lithium cations can store an appropriate amount of hydrogen that has been determined for applied uses under environmental conditions. Researchers are working to create such structures to transform the energy storage process in the near future.

\footnotetext{
${ }^{1}$ MSc Student of Nano Technology, School of new technologies, Iran University of Science and Technology, Tehran, Iran. ${ }^{2}$ Assistant Professor, School of New Technologies, Iran University of Science and Technology, Tehran, Iran. ${ }^{3}$ Assistant Professor, School of New Technologies, Iran University of Science and Technology, Tehran, Iran. ${ }^{4}$ Assistant Professor, Mechanical Engineering department, Semnan University, Semnan, Iran. Correspondence and requests for materials should be addressed to S.S. (email: sadeghzadeh@iust.ac.ir)
} 
One of the techniques that can be used to connect graphene sheets is the irradiation technique, which has already been applied in theory and in practice to connect carbon nanotubes ${ }^{9-11}$. In 2014, Wu et al., In a study conducted by the molecular dynamics simulation technique, introduced a method called "Nano joining of graphene sheets by ion beam irradiation". They showed that two overlapping graphene sheets can connect with the carbon ion beam. The two mechanisms observed for this connection, have been introduced the formation of coordination deflection and the trapping of ions between the plates. Among the most influential parameters on the binding properties, are beam energy and irradiation dose. Optimizing theses can results in better mechanical properties of the binding region than pure graphene. The optimum energy level and the dose of the beam found to be $40 \mathrm{eV}$ and $1.06 \times 10^{15} \mathrm{~cm}^{2}$ respectively ${ }^{12}$. In a similar simulation, argon beam is also used to joining graphene sheets. In this study the mechanism has been proposed as a main factor for the connection, is the creation of dangling bonds during the irradiation process. The tendency of these bonds to saturate and atomic reorganization in the area of the defect causes the formation of the covalent bonds between the sheets. It has been proven that the temperature increase is a beneficial factor that accelerates the saturation process and develops the process of reorganization. In addition, in order to emphasize on the role of irradiation, another experiment was conducted in which the possibility of graphene joining has been studied by applying a large force above the sheets instead of irradiation, and it has been shown that without the irradiation it isn't possible to join the graphene sheets. In the end, the graphene plane's chirality effect on the bonding process has been studied, and it has been determined that the plane with the same chirality result in a better connection ${ }^{13}$. In another study, the effect of focused ion beam irradiation on the graphene stack has also been investigated. It has been observed that a focused ion beam can form covalent bonds between graphene sheets in the stack. In the case of the mechanism of the phenomenon, it is stated that under the irradiation process, large amounts of carbon atoms are removed from the surface of the first layer. By continuing the process of irradiation, carbon atoms with dangling bonds brought around the nanopore, these carbon atoms tend to saturated with other carbon atoms. Thus, the saturation of carbon atoms with dangling bonds in different layers leads to the formation of cross-link between different layers ${ }^{14}$. In 2017 , Wu et al. tried to actually test the joining of graphene sheets by ion irradiation. they used $40 \mathrm{eV}$ of nitrogen ion for this purpose, and then used Raman spectroscopy characterization and atomic force microscopy to test the bonding of the layers, the results indicate the probability of connecting the pages in the overlap area ${ }^{15}$.

In this study, by means of molecular dynamics simulation and exploiting the idea of bonding the layers by the irradiation process and the controlling of the distance between the graphene layers by moisture content, a method for constructing three-dimensional structures of graphene using focused ion beam irradiation proposed. The irradiation of the graphene structure in addition to creating bonds between the individual planes, also can cause the connection between graphene stacks in the border regions. The strength of this three-dimensional structure perpendicular to the graphene sheets is calculated with a range from about 4 to $6 \mathrm{GPa}$, with respect to the diameter of the beam, which is significant in comparison with other substances. It seems that the use of this technique along with the method of controlling the distance between graphene layers by moisture content, pillared structures of graphene can be obtained with an appropriate strength, which can have many applications in the fields of water and energy. This structure is a suitable candidate for use in membrane filtration systems for water $^{16}$, storage and absorption of gas such as hydrogen and carbon dioxide ${ }^{17-20}$, lithium-ion batteries ${ }^{21}$, catalytic processes $^{22}$ and adsorbents ${ }^{23}$.

\section{Materials and Methods}

All simulations of this study were done with the help of large-scale atomic/molecular massively parallel simulator (LAMMPS) software package ${ }^{24}$. Also, all images were made by Ovito software ${ }^{25}$, and all manipulations and modifications to the structures were done with this software too. The graphene building was created with the commands related to the custom lattice. The graphene lattice was defined in such a way that the distance between the carbon atoms in the graphene honeycomb structure is equal to $1.42 \AA^{26}$. The distance between the planes in the base state of the multi-layered graphene structure was $3.4 \AA$, and the graphene plane dimensions in all simulations were $3 \times 3 \mathrm{~nm}$.

In the ion beam irradiation simulation, the silicon element was selected as an ion particle ${ }^{27}$. The energy of the collision particle was set to $40 \mathrm{eV}^{12}$. Due to the high kinetics of the system and preventing system failure and explosion, the system time step was set to $0.1 \mathrm{fs}$. Due to the excessive temperature rise in the system during ion irradiation, graphene temperature was set up by a nose hoover thermostat algorithm in NVT ensemble at $300 \mathrm{~K}$ and the temperature damping parameter was 100 times of the time step. The microcanonical ensemble (NVE) was used for ion beam, otherwise, the velocity of the particles would be overshadowed and diminished. The potential used for this simulation is a hybrid potential of $\mathrm{CH}$-Airebo ${ }^{28}$ and Tersoff- $\mathrm{ZBL}^{29}$ potentials. The Airebo potential models the formation and failure of covalent bonds between carbon atoms in the graphene structure, and the Tersoff-ZBL potential also models the interaction between silicon ions and graphene atoms ${ }^{27}$. The angle of the collision is 90 degrees to the graphene plane and it is perpendicular to the sheets.

Atoms around the layers were fixed to prevent the movement of the layers and the collapse of the initial spacing of the planes during the irradiation process. Applied ensemble to the fixed atoms was NVE. In (Fig. 1), a schematic illustration of the simulation is shown.

The total time of the simulation process was 1.4 million time steps. Prior to the ion collision, graphene was placed at a temperature of $300 \mathrm{~K}$ for 200,000 time steps or 20 picoseconds in order to stabilize the original structure in the NVT ensemble. The total amount of time that graphene exposed to the beam was 100 ps. The total dose of collision ions was considered to be 1,000 to ensure the formation and completion of the pore within the graphene structure. Also, between every two collisions, 1000 time steps were taken to give the system an opportunity to be stabled. Finally, after completing the collision process, before exporting the data from the configuration, the graphene structure for the next $20 \mathrm{ps}$ was placed in the NVT ensemble to stabilize, then the configuration data file was extracted for loading in tensile mechanical testing. The irradiation simulation according to the nature of 


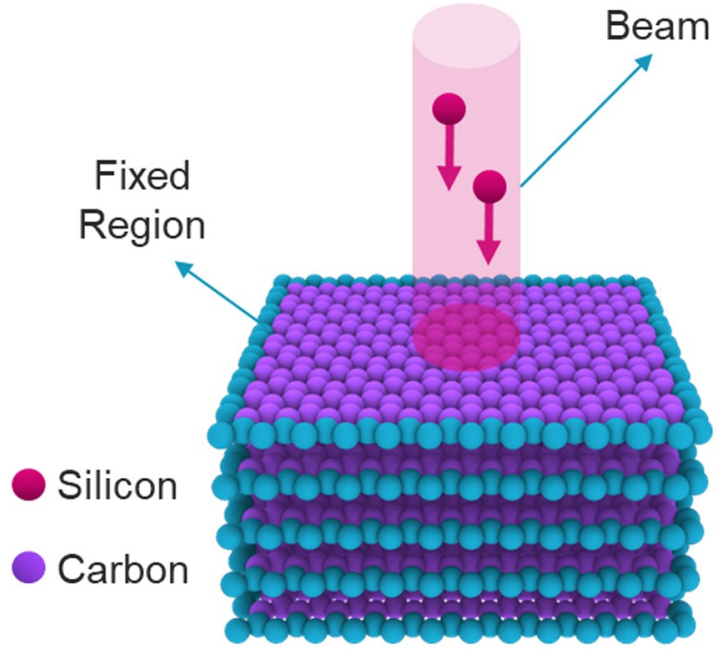

Figure 1. A schematic image of the irradiation process on the graphene layers stack.

the random collisions of particles was repeated for 5 times, in order to increase the accuracy of the measurements in the same conditions, in the mechanical test by averaging the data.

To investigate the mechanical properties of the structure, the strain-stress test was carried out in two directions, perpendicular to the graphene sheets in the direction of the z-vector and parallel to the graphene sheets along the $\mathrm{x}$-vector. The potential used in stress and strain test is Airebo potential. In various researches, in order to correct the potential, the amount of minimum cut-off radius has been modified ${ }^{26,30-34}$. Here also, this value is changed from 1.72 to $2 \AA$. It was observed that the selection of values other than $2 \AA$ results in discrepancies in the results and plots of strain-stress testing in different time steps. By correction of the minimum cut-off radius, the problem of non-conformance in the data was solved. The time step for mechanical simulation was selected to $0.1 \mathrm{fs}$. The strain rate in order to accelerate the simulation process was selected to $0.01 / \mathrm{ps}$. The high strain rate causes a difference of less than $5 \%$ in the final graph, but the simulation speed increases by about ten times. In all strain-stress test simulations, before starting the stretching process, the structure in order to stabilize placed in an NVT ensemble at a temperature of $300 \mathrm{~K}$ for $20 \mathrm{ps}$. The output data extraction rate was every 1000 time steps. Also, as mentioned earlier, each of the graphs is the result of averaging of five tests in completely identical conditions to ensure the accuracy of the results. In the stress calculations, due to the irregular geometry created after the irradiation process, the atomic volume of carbon $\left(8.789 \AA^{3}\right)$ and its multiplication in the number of atoms are used to compute the total system volume.

\section{Results and Discussions}

To investigate the effect of irradiation on a multi-layered graphene sheet, 18 different states were investigated, which has been expressed by three main parameters. These three main parameters are: beam energy, beam diameter and the distance between the graphene layers. For the ion beam, two energy levels of $40 \mathrm{eV}$ and $160 \mathrm{eV}$ were considered to study the effect of increasing energy or velocity of particles, on the formation of covalent bonds between graphene layers. The next parameter is the diameter of the beam, for this quantity three different sizes were considered: $0.5 \mathrm{~nm}, 1.0 \mathrm{~nm}$, and $1.5 \mathrm{~nm}$. The last parameter is the distance between the graphene layers. This distance also has three sizes of $0.34 \mathrm{~nm}, 0.65 \mathrm{~nm}$ and $0.90 \mathrm{~nm}$.

An overview of the results shows that the increase in the beam energy from 40 to 160 electron volts has no considerable effect on the final strengths. Increasing the beam diameter from 0.5 to $1.5 \mathrm{~nm}$ and the gap from 0.34 to 0.65 increases the strength from 50 to $100 \%$, respectively. Increasing the gap from 0.34 and $0.9 \mathrm{~nm}$, for all three sizes of pores, reduces the strength of about $33 \%$. Details are presented in the following sections.

In Fig. 2, all of the 18 structures created after the irradiation process are compared with each other. According to the output images, it appears that between three expressed parameters, the first one, namely beam energy, have the least effect on the final structure, and the difference between the two structures created by different levels of energy is not tangible. In the case of the second parameter, or the diameter of the beam, an increase in the diameter from $0.5 \mathrm{~nm}$ to $1.5 \mathrm{~nm}$ has a significant effect on the increase in the volume of the intermediate links between the graphene sheets. In structures with a small collision diameter, there is no bond between layers. Also, in medium and large diameters, there are layers that are bond-free. And with the increase of the third parameter, the distance between the layers, we see that the density of the bonds is reduced, and short bonds have been converted to long chains between graphene layers. In brief, by increasing the size of the diameter of the beam, the number of covalent bonds increases, and vice versa, with the increase in the distance between the layers, the volume and density of the transverse bonds decreases, and in the meantime, the change in the energy of collision particle has had the least effect, and has not had a visible effect on the density of transverse bonds.

Normal tensile test. In the next step, in order to investigate the strength, each of the structures was separately subjected to a mechanical tensile test. The test was carried out in the direction of the $\mathrm{z}$ vector (perpendicular to the graphene sheets) to evaluate the effect of the formation of cross-links between the graphene layers. 


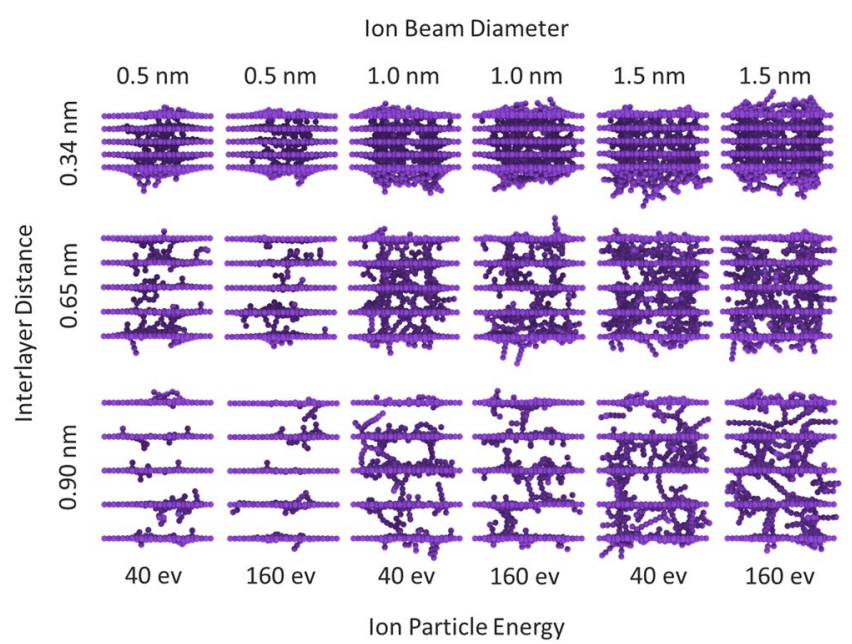

Figure 2. Image of all configurations created after the irradiation process on five-layer stacks of the graphene sheet. The upper axis represents the diameter of the beam, the left axis shows the distance between the layers in the stack and the lower axis indicates the energy of the used beam.

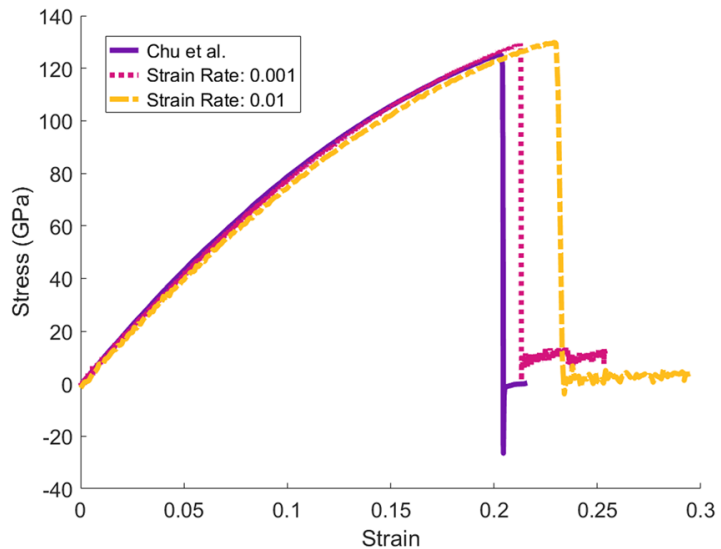

Figure 3. Comparison of SLG strain-stress graphs. The solid line belongs to the reference report ${ }^{32}$. The dotted line is the SLG strain-stress plot at the strain rate of $0.001 / \mathrm{ps}$, and the dashed line is the strain-stress plot of the SLG at the strain rate of $0.01 / \mathrm{ps}$.

Before the process, a strain-stress test was conducted in the "zigzag" direction of the single layer graphene (SLG) sheet in order to validate and compare the output chart in this test with a result from a similar study by Chu et al. as a reference ${ }^{32}$ (Fig. 3). In the study, the minimum cut-off radius in Airebo potential has been modified by the value of $2 \AA$, also the strain rate was equal to $0.001 / \mathrm{ps}$, and the time step value was $0.1 \mathrm{fs}$. In the same conditions, there is a slight difference between the strain-stress plot of the reference test and the chart obtained in this study, this slight difference can be due to the difference in the size of the two graphene. The reference graph refers to graphene with dimensions of $4 \times 4 \mathrm{~nm}$, but in the present test, the graphene dimension is $3 \times 3 \mathrm{~nm}$.

In all next strain-stress tests, the strain rate was set to 0.01/ps. As shown in Fig. 3, the increase in the strain rate caused a slight displacement in the diagram and increased the failure strain by about $8 \%$. The reason for choosing a higher rate of the strain was increasing the speed of simulations, and reducing the time of the test process. By changing the strain rate from $0.001 / \mathrm{ps}$ to $0.01 / \mathrm{ps}$, the simulation speed increases by about 10 times.

As previously stated, by changing the irradiation parameters, states are created in which the planes have no connection with each other, or at least there is no bond between the two graphene layers. In this study, all states with at least two non-bonded sheets lacked the necessary requirements of the strain test and therefore no mechanical test was performed on them. These states are depicted in Fig. 4 as "No Data".

In Fig. 4 it can be seen that as the distance between the graphene layers increases, the ultimate tensile strength in the graphs is reduced, and on the other hand, this value has been increased by increasing the diameter of the collision beam. This quantity has been raised from $4 \mathrm{GPa}$ for a beam with a diameter of $0.5 \mathrm{~nm}$ to about $6 \mathrm{GPa}$ for a beam with a diameter of $1.5 \mathrm{~nm}$. The reason for this increase, as seen in the image of the structures formed after irradiation (Fig. 2), is the density of the bonds formed between the layers. In fact, as the diameter of the beam increases, more carbon atoms are separated from the higher layers and transmitted through the interlayer thereby providing the necessary material to create higher density bonds with a longer chain length. The other parameter, 


\section{Ion Beam Diameter}

$0.5 \mathrm{~nm}$
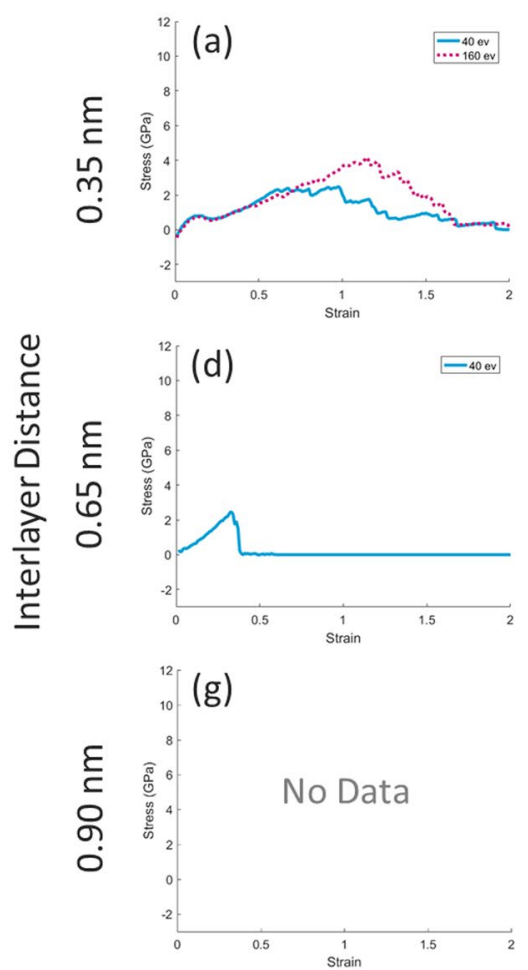

$1.0 \mathrm{~nm}$
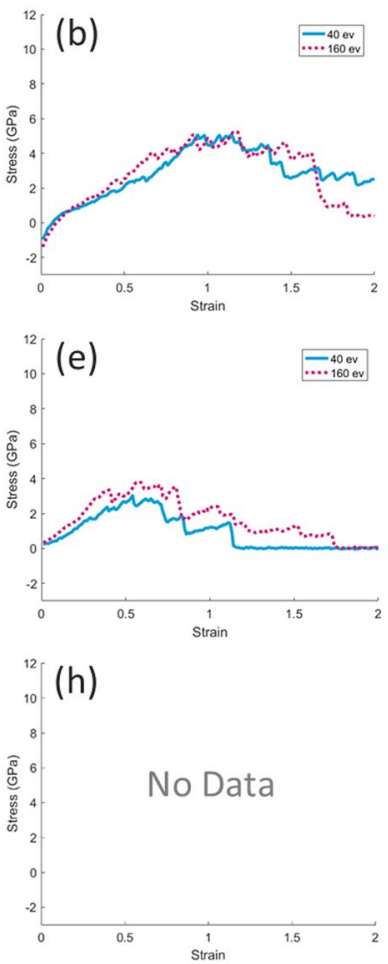

$1.5 \mathrm{~nm}$

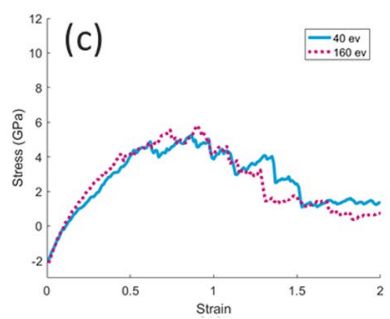

(f)
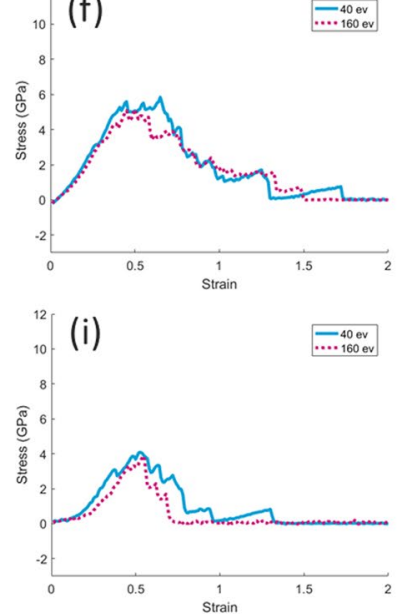

Figure 4. Strain-stress plots of graphene stacks. The blue diagram is related to the configurations generated in the irradiation process at an energy level of $40 \mathrm{eV}$ and the magenta diagram is related to the configurations generated in the irradiation process at an energy level of $160 \mathrm{eV}$. The top axis represents the diameter of the beam used, and the left axis represents the distance between the graphene layers in the stack. (Each of the graphs results from the average of five strain-stress tests on the five configurations created under equal conditions. Situations that do not meet the requirements for the strain-stress test are displayed as "No Data").

ion beam energy, does not have a significant effect on the results, so that in some cases it is seen that the energy of the collision increases the strength and in some cases, in reverse, it reduces the strength. This difference, more than it is related to the beam energy, seems to be due to system fluctuations and collisions and randomized events due to ion collisions on graphene sheets. Although the results were averaging of five separate tests with identical conditions, it should be noted that in the case of (Fig. 4d) at the energy level of 40 electron volts and (Fig. 4i) at the energy level of $160 \mathrm{eV}$, the results obtained are related to only one configuration and the two configurations respectively which had the necessary conditions for the tensile test. Other configurations have been excluded from the mechanical test due to the lack of links at least between two layers of graphene. It seems that increasing the beam energy at higher interlayer distances, increases the risk of no bond formation so that in the state (d), none of the five configurations obtained after the irradiation process with an energy of $160 \mathrm{eV}$ have had a complete connection. But at the energy level of $40 \mathrm{eV}$, of five configurations, in one case a complete connection has occurred. Also in the state (i) for $160 \mathrm{eV}$, of five configurations, only two complete connections have been made, while, in the same conditions for the $40 \mathrm{eV}$ energy level, all five configurations have had a full connection.

As is observable in Fig. 4, the initial value of stress for some cases is negative whereas it should be 0. Prior to performing all stress-strain tests, all structures were relaxed in a constant pressure ensemble for 50 Ps. In addition, to ensure complete relaxation, the energy minimization algorithm, as well as the relaxation of the simulation box, is restored. Such an approach allows both the energy of the atoms at the final configuration to be minimized and the pressure of the system be close to the external pressure (which is zero here). In fact, the reason for the negative tension in the graphs above is the compulsion to maintain the upper and lower graphene sheets during the process of relaxation. This is depicted in Fig. 5 clearly.

Transverse tensile test. For comparison, two other tensile tests were performed in addition to the previous studies. In the first test, pure multilayer non-exposure graphene was subjected to mechanical testing, in order to compare the physical force between the graphene sheets and the states in which the sheets formed a covalent bond (Fig. 6a). In this case, the number of layers was equal to 5 , and the test was performed in the $\mathrm{z}$ vector direction (perpendicular to graphene stack) in the same conditions as previous experiments. In this case, the ultimate tensile strength is equal to $1.17 \mathrm{GPa}$ and the two upper layers of the graphene stack are separated in the strain of 0.16 (Fig. 6b). In the second test, the number of graphene layers was considered to be ten, to evaluate the process 


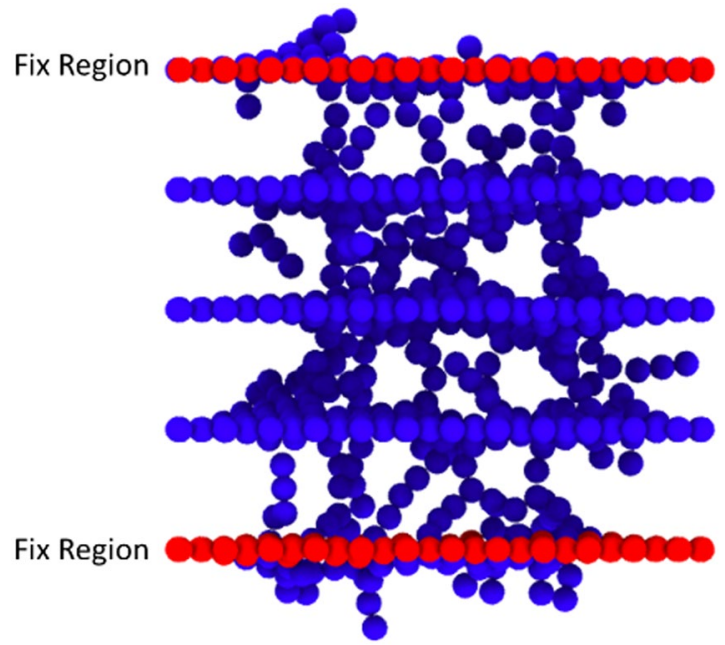

Figure 5. The reason for the negative tension in the stress-strain curves is the compulsion to maintain the upper and lower graphene sheets during the process of relaxation.

(a)

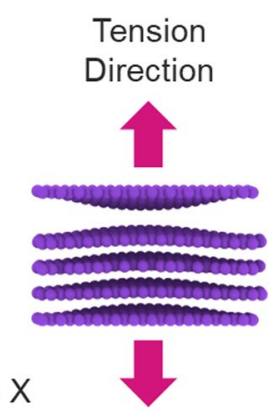

Tension

Direction

(c)

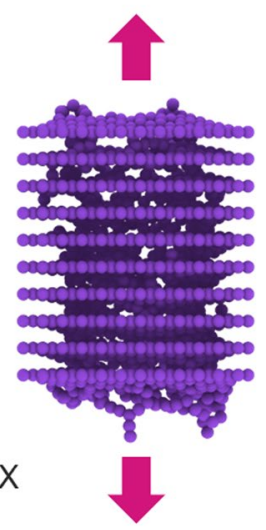

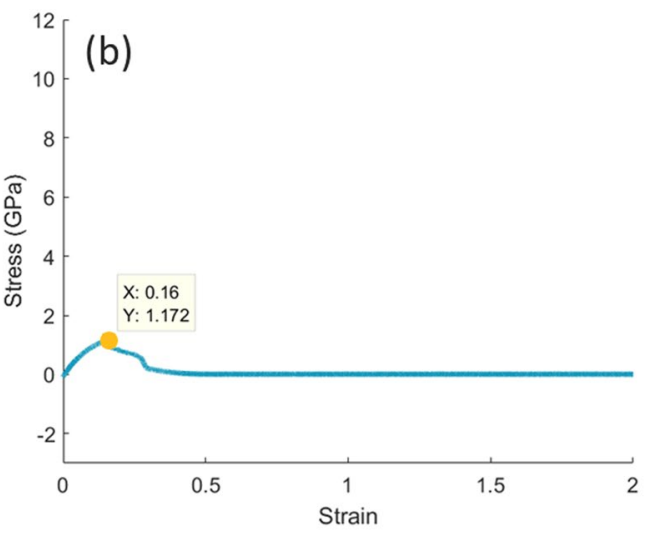

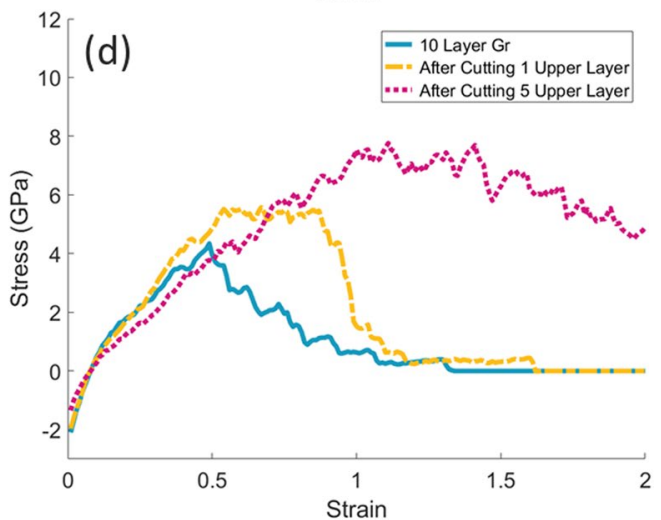

Figure 6. (a) Fracture point of interlayer Van der Waals interaction in the multilayer graphene sheet. (b) Strainstress diagram of multilayer graphene sheet when is under loading perpendicular to sheets. (c) Graphene stack with 10 layers after the irradiation process and forming covalent bonds. (d) Tension test diagram of a 10-layer stack of graphene, the solid line belongs to a 10-layer stack. The dashed line is the result of the tensile test after removal of one upper layer of the stack. The dotted line is the result of strain-stress test after removal of the five upper layers. (All plots are averaged of five strain-stress tests on five different configurations created with equal conditions).

of the nanopore fabrication and subsequently cross-linking in more number of layers (Fig. 6c). The diameter of the beam in this test was $1.0 \mathrm{~nm}$ and the particle energy was considered to be $40 \mathrm{eV}$. The tensile test was also carried out for the ten-layer graphene in the $\mathrm{z}$-direction vector with the same conditions as before. The ultimate 
(a)

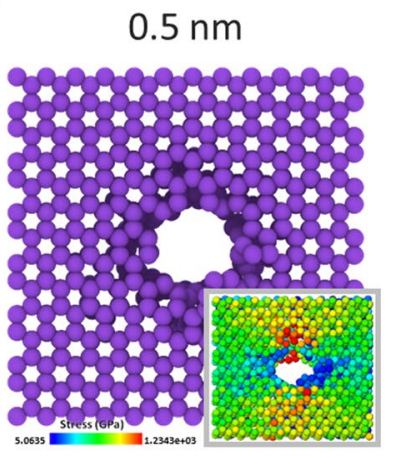

\section{Pore Diameter}
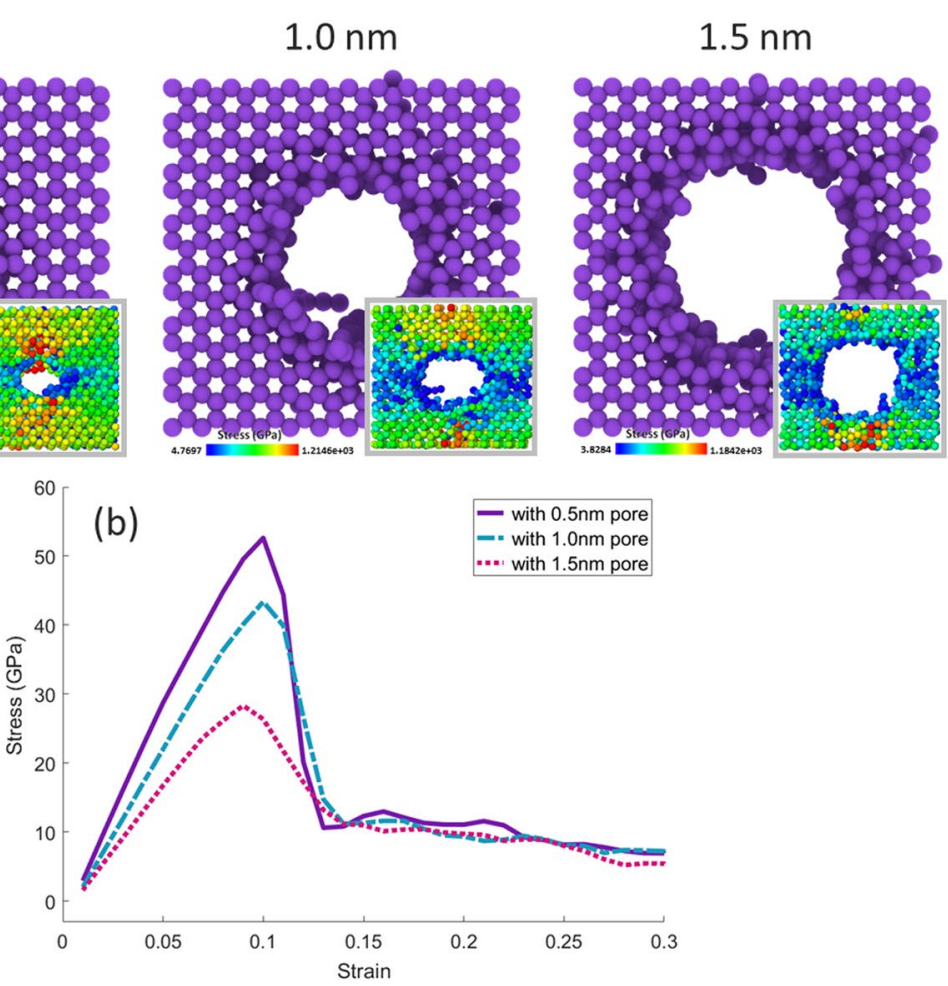

Figure 7. (a) Image of pores created with different diameters on a five-layer stack of graphene sheets. (inset) Tension contour image at the point of ultimate tensile strength associated with each configuration. (b) The diagram of the strain-stress tests in $\mathrm{x}$ vector direction for the configurations shown in (a). The solid curve is the test on the graphene stack with a $0.5 \mathrm{~nm}$ pore, the dashed curve is the test on the graphene stack with a $1.0 \mathrm{~nm}$ pore, and the dotted line is the test on the graphene stack with a $1.5 \mathrm{~nm}$ pore.

tensile strength for ten-layer graphene was obtained about $4 \mathrm{GPa}$ by averaging five separate tests. As shown in Fig. 6c, the lower layers contain more density of links, the reason it seems to be a transferring of carbon atoms from the upper layers to the lower layers. Indeed, the lower layers receive more carbon atoms from the higher layers, which increases the probability of bonding between the underlying layers. The tensile test was again carried out by removing one and five upper layers of graphene stacks respectively to verify graphene strength in these conditions. As shown in the diagram (Fig. 6d), the removal of the first layer with low bonds density improved the graphene strength by about $40 \%$, also removal of the five upper layers has increased the ultimate tensile strength from about $4 \mathrm{GPa}$ to about $8 \mathrm{GPa}$, which indicates the more density and strength of bonds in deeper layers of graphene than the surface layers. In addition, we can see that the area of plastic deformation has expanded dramatically by removing the upper layers.

However, the formation of pores by irradiation process creates bonds between the graphene sheets and reinforces the strength perpendicular to the sheets, in reverse, presence of these pores can weaken the strength in a parallel direction with the sheets. In order to investigate how to change the amount of ultimate tensile strength by changing the size of the pores, three configurations with $0.5 \mathrm{~nm}, 1.0 \mathrm{~nm}$, and $1.5 \mathrm{~nm}$ pore diameter, and a spacing of $0.34 \mathrm{~nm}$, were selected from the configurations obtained during the irradiation process, and The strain-stress test was performed for them in the direction of the x vector "zigzag". As can be seen in the diagram (Fig. 7), the increase in the size of the pore significantly reduced the graphene strength along the $\mathrm{x}$ vector, so that by increasing the size of the pore from a diameter of $0.5 \mathrm{~nm}$ to $1.0 \mathrm{~nm}$, about $20 \%$ of the graphene strength is reduced, and increasing the diameter of the cavity from $1.0 \mathrm{~nm}$ to $1.5 \mathrm{~nm}$ resulted in a sharp reduction (about $60 \%$ ) in the strength. However, in the worst case, graphene with a 1.5-nanometer cavity, the ultimate tensile strength is about $30 \mathrm{GPa}$, which is far superior to many other materials.

Nano welding. In addition to all the tests carried out, the irradiation process for joining and strengthening, in which two stacks of graphene were flanked side by side, were also examined. A graphene film contains large boundary regions which are formed by adjoining graphene sheets and stacks. The study of the effect of the ion beam on these areas can also be important. For this purpose, with the same conditions as previous, a similar test was conducted in the boundary areas between the two five-layer stacks of the graphene. Variable parameters in this test are the diameter of the beam, the spacing of the layers and the boundary size between two graphene stacks. The diameter of the beam for this test was $1.0 \mathrm{~nm}$ and $1.5 \mathrm{~nm}$. Sheets in the stack were placed at a distance of 0.34 and $0.65 \mathrm{~nm}$ to each other, and the size of the boundary regions between the two stacks of graphene were 
Ion Beam Diameter

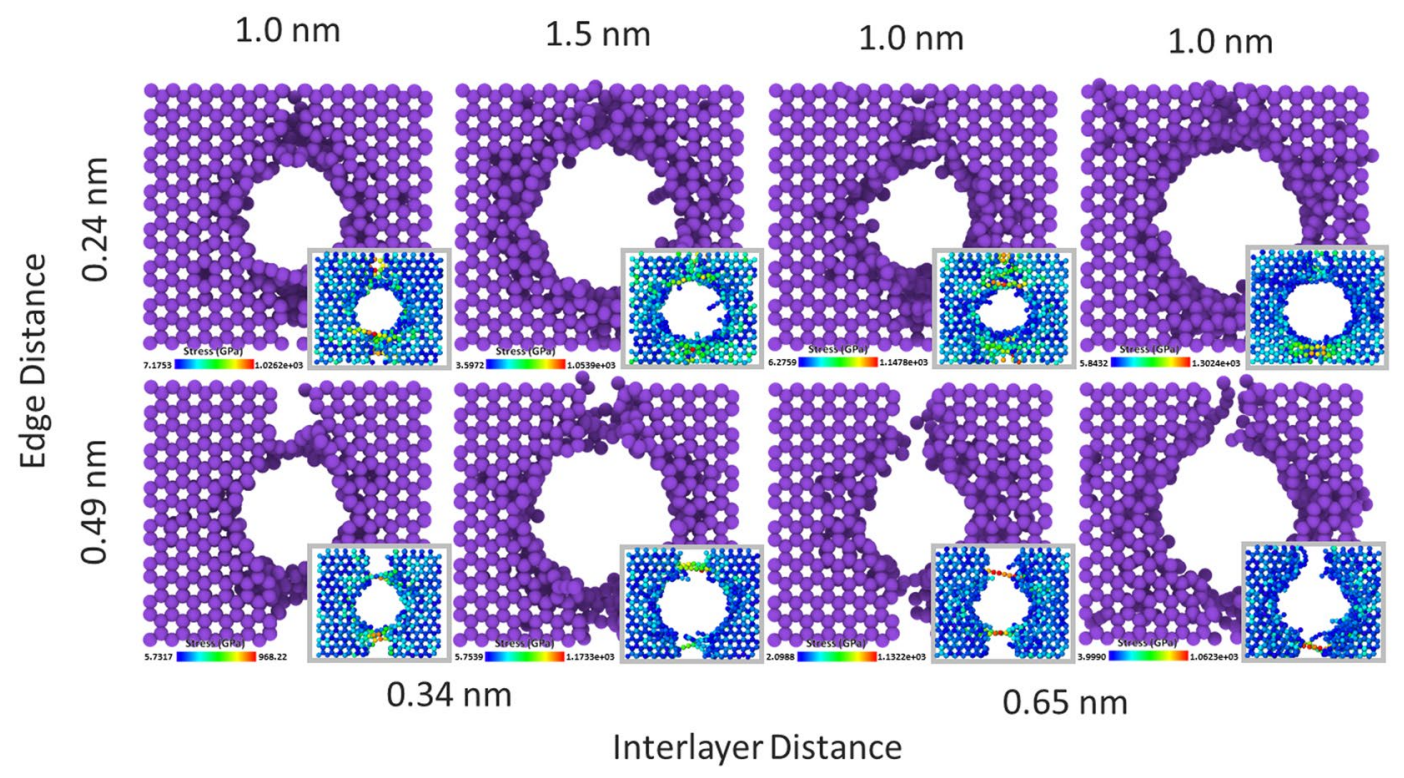

Figure 8. Configurations obtained after the irradiation process in the boundary region between two five-layer stacks of graphene. (inset) Tension contour at the point of ultimate tensile strength.
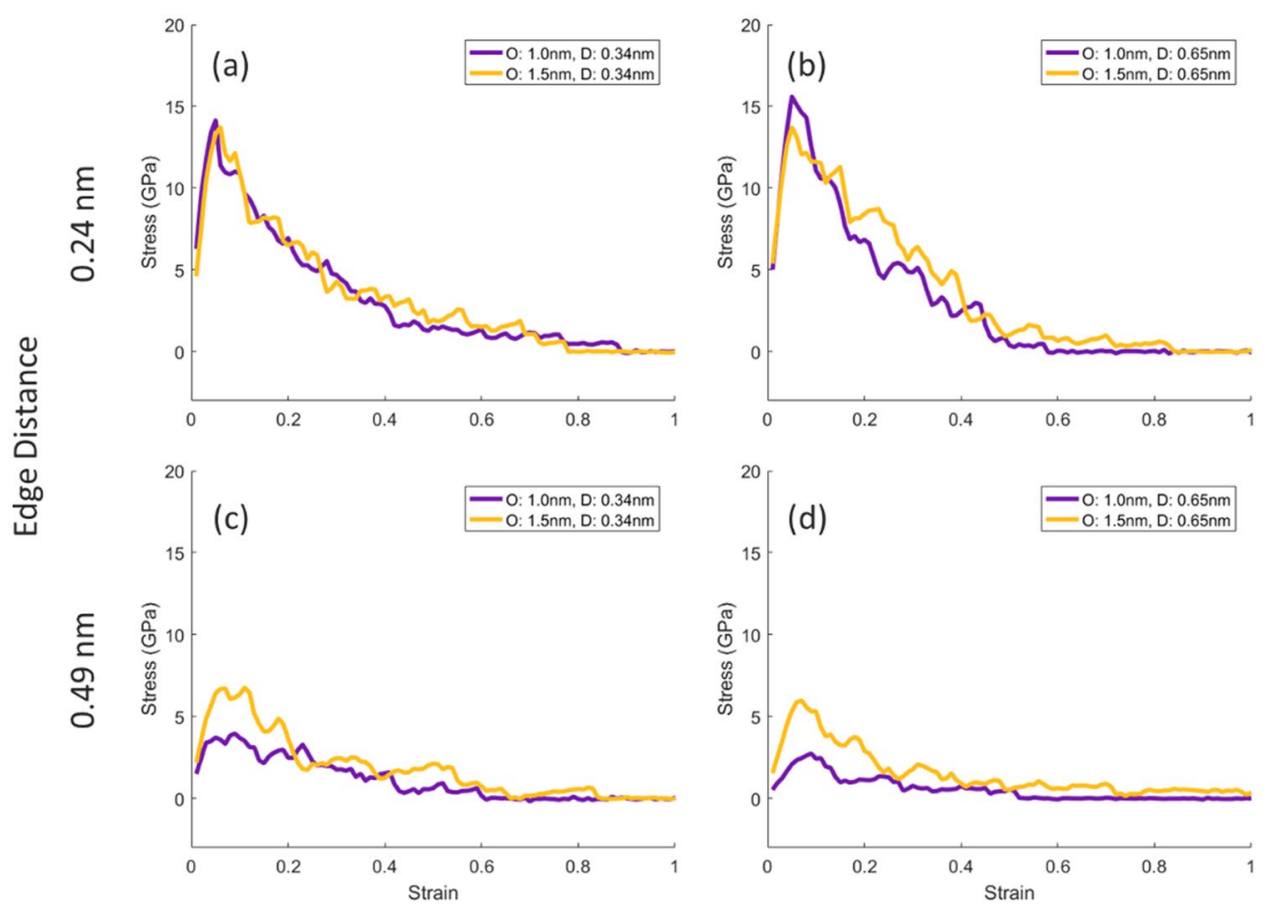

Figure 9. (a-d) Graphs obtained from the strain-stress test in the direction of $\mathrm{x}$ vector "zigzag" for the joined stacks of graphene in the boundary area shown in Fig. 8.

$0.24 \mathrm{~nm}$ and $0.49 \mathrm{~nm}$ which were created by removing one row and two rows of carbon atoms from the integrated graphene structure, respectively (Fig. 8).

According to the graphs obtained from the strain-stress test on the eight existing states (Fig. 9), increase in the distance between two graphene stacks, namely the size of the boundary area, has the greatest effect on the ultimate tensile strength. By increasing the boundary size from $0.24 \mathrm{~nm}$ to $0.49 \mathrm{~nm}$, the ultimate tensile strength has been halved, and this decrease is seen regardless of the distance between the graphene layers and the size of the pore in each of the eight states. Increasing the diameter of the beam from $1.0 \mathrm{~nm}$ to $1.5 \mathrm{~nm}$, with a boundary size of $0.24 \mathrm{~nm}$, has not had a significant effect on the graph, but in the case of a $0.49 \mathrm{~nm}$ of boundary size, the 
(a) 2

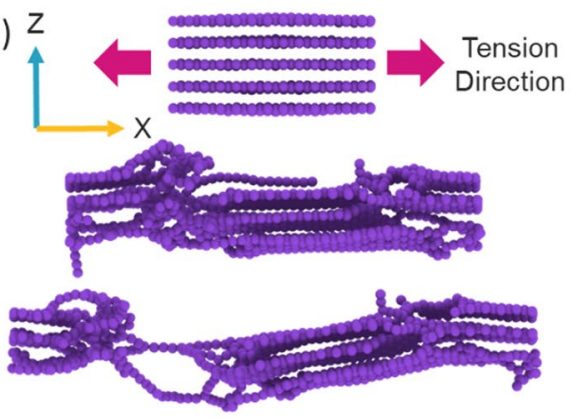

(c) $z$

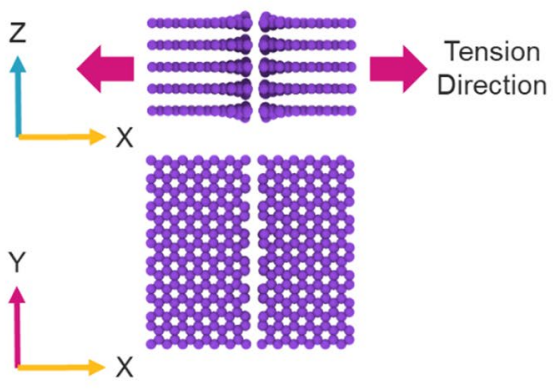

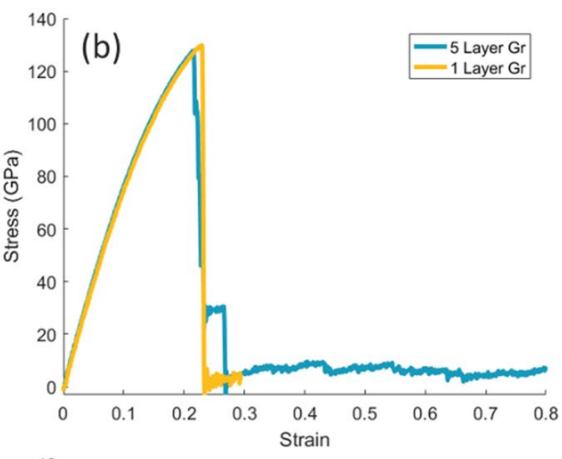

(d)

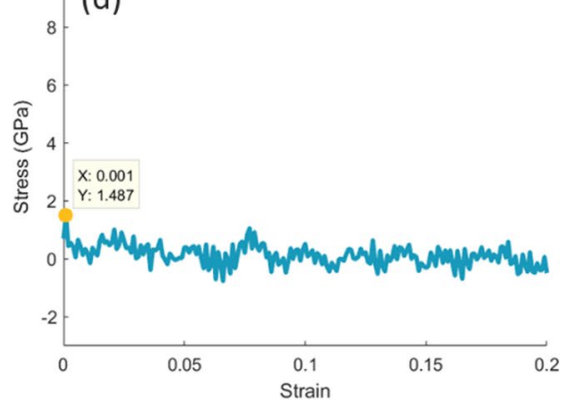

Figure 10. (a) A simulation image of a tensile test on a five-layer stack of graphene sheets in a zigzag direction. (b) Comparison of strain-stress test on SLG and five-layer graphene stack in a zigzag direction. (The yellow curve is related to SLG and the blue curve is related to the five-layer graphene stack). (c) Image of two separate graphene stacks. (d) Strain-stress test diagrams for two separate graphene stacks.

increase in beam diameter contributed to the increase in strength and left a notable increase in the strain-stress curve. Meanwhile, the change in the distance between the graphene sheets has had the least effect on the ultimate tensile strength. Each of the graphs is averaged over five strain-stress tests on five different configurations created in equal conditions.

For comparison, a pure five-layer graphene stack without porosity was subjected to the tensile test (Fig. 10a). The ultimate tensile strength of this structure was $127 \mathrm{GPa}$, which is about $1.7 \%$ less than the SLG. The amount of the stress in this structure, even after applying a strain of 0.8 , has not been reduced to zero, due to the tearing and sliding and the reformation of the bonds between the various layers, which has created a wide area of plastic deformation. In the next, one row of the carbon atoms was removed from the middle of the sheets and then the structure was loaded without performing the irradiation process. In this case, the amount of interaction between two graphene stacks is so small that it cannot be detected by system fluctuations. However, the highest tensile stress recorded is in the strain of 0.001 and it is about $1.5 \mathrm{GPA}$. Therefore, in the best case, the irradiation process improves the ultimate tensile strength by $1000 \%$ in a boundary area between two graphene stacks, which is about one-tenth of the integrated graphene structure. In the worst case, i.e. when the distance between the two stacks is $0.49 \AA$ and the beam diameter is equal to $1 \mathrm{~nm}$, we also see a $2.5 \mathrm{GPa}$ strength in the boundary area after the irradiation process, which is about one-fifth of the ultimate tensile strength in the integrated graphene structure.

\section{Conclusion}

Bonding graphene layers by irradiation process and controlling of the distance between the graphene layers by moisture content, a method for constructing three-dimensional graphene structures was proposed. By presenting this new idea, the following results are achieved:

1. Ion beam energy, have the least effect on the final structure, and the difference between the two structures created by different levels of energy is not tangible. An increase in the diameter from $0.5 \mathrm{~nm}$ to $1.5 \mathrm{~nm}$ has a significant effect on the increase in the volume of the intermediate links between the graphene sheets.

2. As the distance between the graphene layers increases, the ultimate tensile strength is reduced and increased by increasing the diameter of the collision beam from $4 \mathrm{GPa}$ for a beam with a diameter of $0.5 \mathrm{~nm}$ to about $6 \mathrm{GPa}$ for a beam with a diameter of $1.5 \mathrm{~nm}$.

3. Performing a transverse tensile test after ion beam irradiation on a ten-layer graphene stack, for all simulations, the lower layers contain more density of links, because of transferring of carbon atoms from the upper layers to the lower layers. Indeed, the lower layers receive more carbon atoms from the higher layers, which increases the probability of bonding between the underlying layers. The removal of the first layer with low bonds density improved the graphene strength by about $40 \%$. Also, removal of the five upper layers has increased the ultimate tensile strength from about $4 \mathrm{GPa}$ to about $8 \mathrm{GPa}$, which indicates the more density and strength of bonds in deeper layers of graphene than the surface layers. 
4. Increasing the size of the pores significantly has reduced the graphene strength so that by increasing the pore size from $0.5 \mathrm{~nm}$ to $1.0 \mathrm{~nm}$, about $20 \%$ of the graphene strength is reduced, and increasing it from $1.0 \mathrm{~nm}$ to $1.5 \mathrm{~nm}$ resulted in a strong reduction (about 60\%) in the strength. However, in the worst case, i.e. porous graphene with a 1.5 -nanometer pore, the ultimate tensile strength is about $30 \mathrm{GPa}$, which is far superior to many other materials.

5. Finally, nano welding of graphene sheets was proposed via ion beam irradiation. By increasing the boundary size from $0.24 \mathrm{~nm}$ to $0.49 \mathrm{~nm}$, the ultimate tensile strength has been halved. Increasing the diameter of the beam from $1.0 \mathrm{~nm}$ to $1.5 \mathrm{~nm}$, with a boundary size of $0.24 \mathrm{~nm}$, has not had a significant effect on the graph, but in the case of a $0.49 \mathrm{~nm}$ of boundary size, the increase in beam diameter contributed to the increase in strength and left a notable increase in the strain-stress curve. Meanwhile, the change in the distance between the graphene sheets has had the least effect on the ultimate tensile strength. As a fantastic result, in the best case, the irradiation process improves the ultimate tensile strength by $1000 \%$ in the boundary area between two graphene stacks, which is about one-tenth of the integrated graphene structure.

\section{References}

1. Burress, J. W. et al. Graphene oxide framework materials: theoretical predictions and experimental results. Angewandte Chemie International Edition 49, 8902-8904 (2010).

2. Hung, W.-S. et al. Cross-linking with diamine monomers to prepare composite graphene oxide-framework membranes with varying d-spacing. Chemistry of Materials 26, 2983-2990 (2014).

3. Hua, D., Rai, R. K., Zhang, Y. \& Chung, T.-S. Aldehyde functionalized graphene oxide frameworks as robust membrane materials for pervaporative alcohol dehydration. Chemical Engineering Science 161, 341-349 (2017).

4. Tsoufis, T. et al. Tailor-made graphite oxide-DAB poly (propylene imine) dendrimer intercalated hybrids and their potential for efficient $\mathrm{CO}_{2}$ adsorption. Chemical Communications 50, 10967-1097 (2014).

5. Kumar, R., Suresh, V. M., Maji, T. K. \& Rao, C. Porous graphene frameworks pillared by organic linkers with tunable surface area and gas storage properties. Chemical Communications 50, 2015-2017 (2014).

6. Feng, B., Xu, K. \& Huang, A. Covalent synthesis of three-dimensional graphene oxide framework (GOF) membrane for seawater desalination. Desalination 394, 123-130 (2016).

7. Abraham, J. et al. Tunable sieving of ions using graphene oxide membranes. Nature nanotechnology 12, 546 (2017).

8. Dimitrakakis, G. K., Tylianakis, E. \& Froudakis, G. E. Pillared graphene: a new 3-D network nanostructure for enhanced hydrogen storage. Nano letters 8, 3166-3170 (2008).

9. Terrones, M. et al. Molecular junctions by joining single-walled carbon nanotubes. Physical review letters 89, 075505 (2002)

10. Krasheninnikov, A., Nordlund, K., Keinonen, J. \& Banhart, F. Making junctions between carbon nanotubes using an ion beam. Nuclear Instruments and Methods in Physics Research Section B: Beam Interactions with Materials and Atoms 202, 224-229 (2003).

11. Wang, Z. et al. Amorphous molecular junctions produced by ion irradiation on carbon nanotubes. Physics Letters A 324, 321-325 (2004).

12. Wu, X., Zhao, H., Zhong, M., Murakawa, H. \& Tsukamoto, M. Molecular dynamics simulation of graphene sheets joining under ion beam irradiation. Carbon 66, 31-38 (2014).

13. $\mathrm{Wu}, \mathrm{X}$., Zhao, H. \& Murakawa, H. The joining of graphene sheets under Ar ion beam irradiation. Journal of nanoscience and nanotechnology 14, 5697-5702 (2014).

14. Wu, X., Zhao, H. \& Pei, J. Fabrication of nanopore in graphene by electron and ion beam irradiation: Influence of graphene thickness and substrate. Computational Materials Science 102, 258-266 (2015).

15. Wu, X., Zhao, H., Pei, J. \& Yan, D. Joining of graphene flakes by low energy N ion beam irradiation. Applied Physics Letters 110, $133102(2017)$.

16. Nicolaï, A., Sumpter, B. G. \& Meunier, V. Tunable water desalination across graphene oxide framework membranes. Physical Chemistry Chemical Physics 16, 8646-8654 (2014).

17. Mercier, G. et al. Porous graphene oxide/diboronic acid materials: structure and hydrogen sorption. The Journal of Physical Chemistry C 119, 27179-27191 (2015).

18. Srinivas, G., Burress, J. W., Ford, J. \& Yildirim, T. Porous graphene oxide frameworks: synthesis and gas sorption properties. Journal of Materials Chemistry 21, 11323-11329 (2011).

19. Chan, Y. \& Hill, J. M. Hydrogen storage inside graphene-oxide frameworks. Nanotechnology 22, 305403 (2011).

20. Haque, E. et al. Boron-Functionalized Graphene Oxide-Organic Frameworks for Highly Efficient $\mathrm{CO}_{2} \mathrm{Capture}_{\text {. Chemistry-An Asian }}$ Journal 12, 283-288 (2017).

21. Nanjundan, A. K. et al. Porphyrin-graphene oxide frameworks for long life sodium ion batteries. Journal of Materials Chemistry Ax (2017).

22. Tran, T. P. N., Thakur, A., Trinh, D. X., Dao, A. T. N. \& Taniike, T. Design of Pd@ Graphene oxide framework nanocatalyst with improved activity and recyclability in Suzuki-Miyaura cross-coupling reaction. Applied. Catalysis A: General 549, 60-67 (2018)

23. Li, M. et al. Graphene oxide framework: an adsorbent for solid phase extraction of phenylurea herbicides from water and celery samples. Journal of Chromatography A 1469, 17-24 (2016).

24. Plimpton, S. Fast parallel algorithms for short-range molecular dynamics. Journal of computational physics 117, 1-19 (1995).

25. Stukowski, A. Visualization and analysis of atomistic simulation data with OVITO-the Open Visualization Tool. Modelling and Simulation in Materials Science and Engineering 18, 015012 (2009).

26. Fu, Y., Ragab, T. \& Basaran, C. The effect of Stone-Wales defects on the mechanical behavior of graphene nano-ribbons. Computational Materials Science 124, 142-150 (2016).

27. Bai, Z., Zhang, L., Li, H. \& Liu, L. Nanopore creation in graphene by ion beam irradiation: geometry, quality, and efficiency. ACS applied materials \& interfaces 8, 24803-24809 (2016).

28. Stuart, S. J., Tutein, A. B. \& Harrison, J. A. A reactive potential for hydrocarbons with intermolecular interactions. The Journal of chemical physics 112, 6472-6486 (2000).

29. Devanathan, R., de la Rubia, T. D. \& Weber, W. Displacement threshold energies in $\beta$-SiC. Journal of nuclear materials 253, 47-52 (1998).

30. Zhao, H., Min, K. \& Aluru, N. Size and chirality dependent elastic properties of graphene nanoribbons under uniaxial tension. Nano letters 9, 3012-3015 (2009).

31. Deng, B. et al. The normal-auxeticity mechanical phase transition in graphene. 2D Materials 4, 021020, https://doi.org/10.1088/20531583/aa61e5 (2017).

32. Chu, Y., Ragab, T. \& Basaran, C. The size effect in mechanical properties of finite-sized graphene nanoribbon. Computational Materials Science 81, 269-274 (2014). 
33. He, L., Guo, S., Lei, J., Sha, Z. \& Liu, Z. The effect of Stone-Thrower-Wales defects on mechanical properties of graphene sheets-A molecular dynamics study. Carbon 75, 124-132 (2014).

34. $\mathrm{Wu}, \mathrm{J}$. \& Wei, Y. Grain misorientation and grain-boundary rotation dependent mechanical properties in polycrystalline graphene. Journal of the Mechanics and Physics of Solids 61, 1421-14 (2013).

\section{Author Contributions}

M.A. Abdol carried out the simulations and wrote the article under supervision of Dr. S. Sadeghzadeh. Dr. M. Jalaly involved in discussion to achieving final plan and Dr. M. M. Khatibi has monitored computing using high performance computers.

\section{Additional Information}

Supplementary information accompanies this paper at https://doi.org/10.1038/s41598-019-44697-z.

Competing Interests: The authors declare no competing interests.

Publisher's note: Springer Nature remains neutral with regard to jurisdictional claims in published maps and institutional affiliations.

(c) (i) Open Access This article is licensed under a Creative Commons Attribution 4.0 International Br License, which permits use, sharing, adaptation, distribution and reproduction in any medium or format, as long as you give appropriate credit to the original author(s) and the source, provide a link to the Creative Commons license, and indicate if changes were made. The images or other third party material in this article are included in the article's Creative Commons license, unless indicated otherwise in a credit line to the material. If material is not included in the article's Creative Commons license and your intended use is not permitted by statutory regulation or exceeds the permitted use, you will need to obtain permission directly from the copyright holder. To view a copy of this license, visit http://creativecommons.org/licenses/by/4.0/.

(C) The Author(s) 2019 